\title{
EXPERIMENTAL METHODS OF THE STUDY OF VORTEX STRUCTURES EXCITED BY POINT INJECTION AT THE LEADING EDGE OF THE OBLIQUE WING
}

\author{
S. N. Tolkachev, V. N. Gorev, G. M. Zharkova, \\ and V.N. Kovrizhina
}

Khristianovich Institute of Theoretical and Applied Mechanics Siberian Branch of the Russian Academy of Sciences 4/1 Institutskaya Str., Novosibirsk 630000, Russia

The paper presents the results of application of thermoanemometry on a curved surface and the liquid crystal thermography (LCT) to study the stability of the flow at the leading edge of an oblique wing. Quantitative results of the distribution of the velocity perturbation in the boundary layer near the attachment line were obtained with the help of thermoanemometry. A decrease of boundary-layer stability because of modification of its structure by stationary vortex is found. The method of LCT allowed to expand the study area to $70^{\circ}$ on both sides of the attachment line, to get pictures of disturbed flow visualization for multiple modes of injection, and to show the influence of the injection velocity on the size and trajectory of stationary disturbances induced by air jet. Visualization results are consistent with the thermoanemometry.

\section{INTRODUCTION}

\subsection{Laminar-Turbulent Transition on the Oblique Wing}

The boundary layer of oblique wing has a three-dimensional structure. There are longitudinal and transverse velocity components which could be separated. Cross-flow velocity profile has an inflection point and thus unstable. Laminarturbulent transition on an oblique wing occurs in the following scenario: in a favorable pressure gradient near the leading edge, in the boundary layer, there appears cross-flow instability $[1,2]$, which has a form of longitudinal vortices

This is an Open Access article distributed under the terms of the Creative Commons Attribution License 4.0, which permits unrestricted use, distribution, and reproduction in any medium, provided the original work is properly cited. 
that modify the mean flow $[3,4]$. As a result, in boundary layer, there appears the region which contains inflection points of velocity profiles. These conditions become favorable for appearance and development of secondary highfrequency disturbances [5]. This process changes the flow regime to the turbulent one.

\subsection{Measurement Techniques at the Leading Edge}

The leading edge of each wing is a curved surface that complicates the boundary layer study. Researchers have different points of view to choose the profile for the experiment. Some of them choose real airfoils during the collaboration with aircraft industry. On the other hand, the flow characteristics will be obtained only for this profile and it becomes difficult to generalize them. Other investigators use a swept cylinder in experiments as a first approximation of any leading edge. To prevent separation on the leeward side, the plane and turbulators were installed.

Another problem is the measurement on the oblique wing leading edge. Hotwire measurements require that the sensor wire should be tangent to the surface and have a possibility for measuring only one component of velocity. Two- and, moreover, three-wire sensors have stronger influence on the flow and significantly increase the measurement volume, which is not acceptable for a thin boundary layer near the attachment line. Researchers have the different views on hotwire technique: some of them use fixed to the surface hot-wire anemometer sensors [6], other use traverse mechanisms [7], the automation of which allows to acquire a large amount of data.

The present authors will also highlight the visualization of the flow as a technique of research of the leading edge boundary layer. Methods of oil-film visualization and naphthalene sublimation themselves are disturbing because of changing the surface roughness, causing the formation of stationary vortices. Besides, the oil-film visualization method has insufficient sensitivity and it is necessary to stop wind tunnel after each test regime.

The LCT method allows the visualization of the distribution of temperature and heat flow which reflects the structure of near-wall flow model $[8,9]$. It is interactive, allowing to change the conditions of the experiment, with almost no influence on the surface roughness, but it can have a thermal effect on the flow.

\subsection{Blowing}

The use of roughness in the experiments on the stability of the boundary layer encounters the next challenge - it is hard to change the size and shape of the 
roughness during the experiment and there is a limited set of testing combinations. In this situation, there is another attractive way to excite stationary vortices - blowing through a small hole. Experiments [10-12] confirm the formation of the pair of stationary vortices in the boundary layer behind a single jet. Because roughness also excites a pair of counterrotating vortices, its effect looks similar to that of the localized blowing. At the same time, blowing is a convenient tool to study the stability of the boundary layer, since it is possible to adjust the blowing velocity, simulating roughness of various sizes. Pneumoroute connected to the speaker allows the speaker to introduce high-frequency disturbances through the hole regardless of the compressor operation.

It should be noted that the physics of the excitation of stationary vortices by roughness and air jets differs. The roughness can be modeled by weak blowing. An increase of the blowing velocity can lead to a complication of the flow pattern.

The aim of this work is to determine the effect of point blowing at the leading edge boundary layer and establish its suitability as a source of disturbances, simulating a single roughness element using an automated hot-wire anemometry and the LCT method.

\section{INVESTIGATION OF THE OBLIQUE WING LEADING EDGE}

\subsection{Measurement Conditions}

Experiment was carried out in a low-turbulent wind tunnel T-324 of the Institute of Theoretical and Applied Mechanics with a cross section of the test section $1000 \times 1000 \mathrm{~mm}$ and a length of $4000 \mathrm{~mm}$. The free-stream turbulence level did not exceed $0.03 \%$. Free-stream velocity through hot-wire measurements was $U_{\infty}=4.2 \mathrm{~m} / \mathrm{s}$, and during the LCT, it was ranged as $U_{\infty}=3.4-$ $9.4 \mathrm{~m} / \mathrm{s}$ and controlled with a help of Pitot-static tube. The air temperature was $22{ }^{\circ} \mathrm{C}$.

In order to improve the quality of visualization, illumination of the wind tunnel test section was supplemented by two halogen lamps with a reflector of $100 \mathrm{~W}$ each. The lamps were positioned so that illumination of the test area at the leading edge of the wing did not give glare and also did not affect the flow in the wind tunnel.

\subsection{Investigated Model}

For investigations of the leading edge flow, an acrylic swept wing model of $5 \mathrm{~mm}$ thick was designed. The chord was $c_{h}=400 \mathrm{~mm}$ and the sweep angle was 


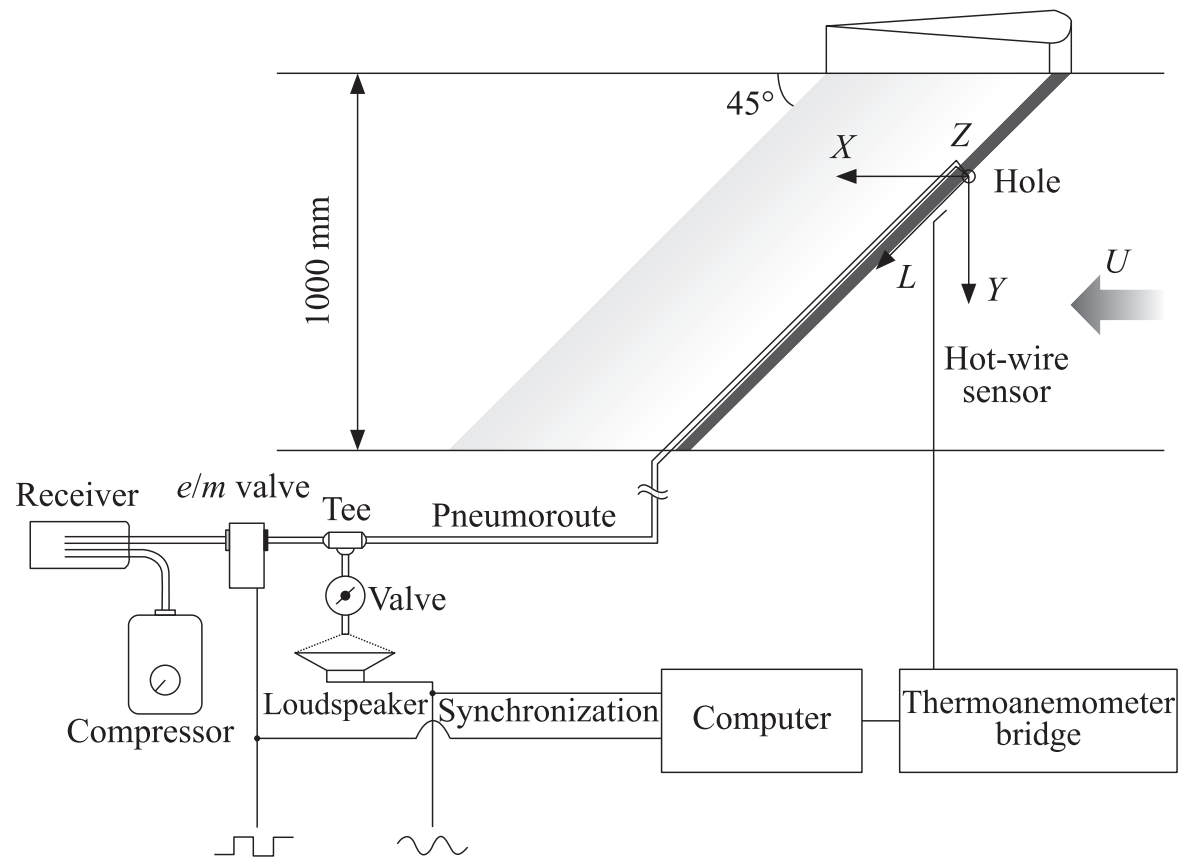

Figure 1 Experimental setup for investigation of the influence of air jet on the flow near the attachment line using hot-wire measurements

$\chi=45^{\circ}$ with a continuously variable angle of attack (Fig. 1). As the model was intended only for research at the leading edge, a simple airfoil was chosen, which was formed by a cylinder and two planes. Physically, the leading edge of each wing is a kind of a cylinder, which allows one to simplify and generalize the results of the experimental measurements for any other profile. To avoid boundary layer separation causing flow pulsation in the upstream region, the turbulators were installed at the maximum thickness of the airfoil (along the boundaries between cylinder and plates). The disturbances developing in the boundary layer of the test section wall were cut off by the endplates.

At the leading edge of the oblique wing, a hole with the diameter $d_{\text {hole }}$ $=0.5 \mathrm{~mm}$ connected to the compressor by pneumoroute was arranged. The hole location was chosen to be at the attachment line at zero angle of attack.

\subsection{Technique of Injecting of Controlled Disturbances}

The disturbance was injected with a help of technique of controlled blowing through the hole (see Fig. 1). Blowing was produced by a compressor, the dura- 
tion was defined by fast electromagnetic valve synchronized with the recording signal.

Valve switching frequency was $0.5 \mathrm{~Hz}$, thus, longitudinal disturbances introduced into the boundary layer were quasi-stationary. The influence of air jet velocity on the flow pattern was investigated. Two regimes of disturbance injection superimposed on the low-frequency blowing were realized: with and without high-frequency component overlapping. High-frequency perturbations of 110,160 , and $210 \mathrm{~Hz}$ were injected into the flow through a tee pipe after electromagnetic valve using a dynamic loudspeaker, according to the method described in $[13,14]$. The only difference was that for the generation of longitudinal disturbance, the compressor was used instead of a loudspeaker (see Fig. 1). By adjusting the angle of attack $\alpha$ near zero, different positions $d$ of the injection point relative to the attachment line were reached, thus, the behavior of the examined disturbances changed.

\subsection{Hot-Wire Measurements}

The test model was positioned horizontally (see Fig. 1). In the coordinate system used in what follows, $X$-axis is directed along the flow, $Z$-axis is vertical and tangent to the wing leading edge, and $Y$-axis is perpendicular to the $X-Z$ plane. The origin of coordinates coincides with the center hole or with the projection of this point on the cylinder axis. Furthermore, we use an additional axis $L$ was used which is directed along the leading edge.

The measurements were carried out by a one-wire probe of a constant temperature anemometer. The diameter of the wire was $6 \mu \mathrm{m}$, its length was about $1 \mathrm{~mm}$. Oscillograms of velocity $U(t)$ at different points in space $(x, y, z)$ were recorded and acquired in a personal computer. Free-stream velocity in the test section of the wind tunnel was measured by Pitot - static tube connected to an electronic micromanometer. The hot-wire probe was calibrated in the free stream against the Pitot - static tube at the free-stream velocity of $0-6 \mathrm{~m} / \mathrm{s}$, so that the error in determining the average speed was less than $2 \%$. The calibration process and the applied experimental equipment are described in detail in $[15]$.

The hot-wire signal was transmitted in the form of velocity oscillograms to the computer via analog-to-digital converter. In order to improve the signalto-noise ratio, ensemble averaging was used. The information about amplitude and phase distributions of the disturbances were received using Fourier transform. Hot-wire data were obtained using two grids of measurement points. The first one was tangent to the attachment line and parallel to the coordinate $Z$ (Fig. $2 a$ ) at different distances from the source of disturbances. The simplicity of these measurements allowed adjustment of the experiment regime to be the optimum one. For the second one (Fig. 2b), a methodology of hot-wire mea- 


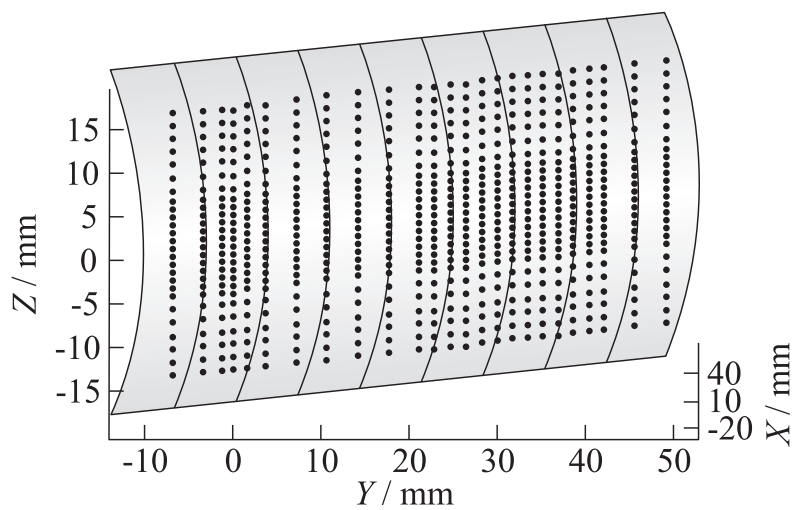

(a)

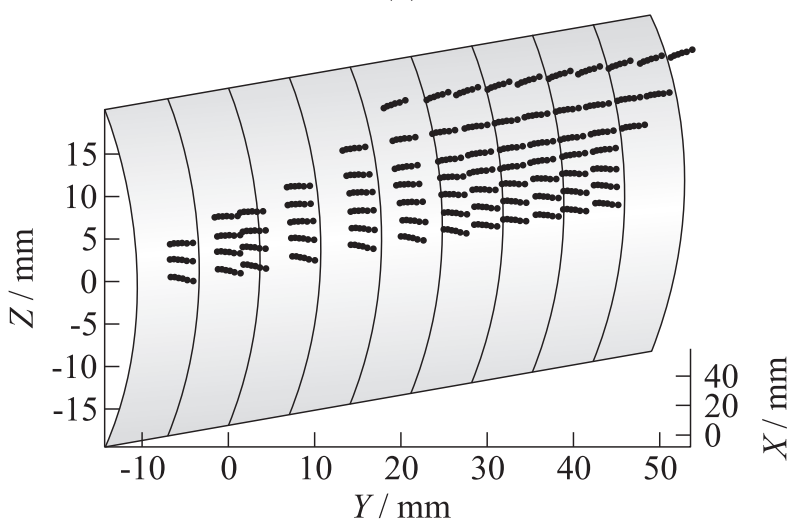

(b)

Figure 2 Hot-wire measurement points on the oblique wing leading edge

surements in the entire boundary layer over the curved surface was developed. For this purpose, the hot-wire probe was rotated around its axis when it passed the angular coordinate to support the tangent orientation of the wire to the surface.

\subsection{Liquid Crystal Thermography Technique}

The heat transfer is proportional to $d U / d y$ where $U$ is the local flow velocity and $y$ is the coordinate normal to the wall. By measuring the heat transfer coefficient at each point, the location of the laminar-turbulent transition, separated flow region, and the location of stationary disturbances can be determined. 


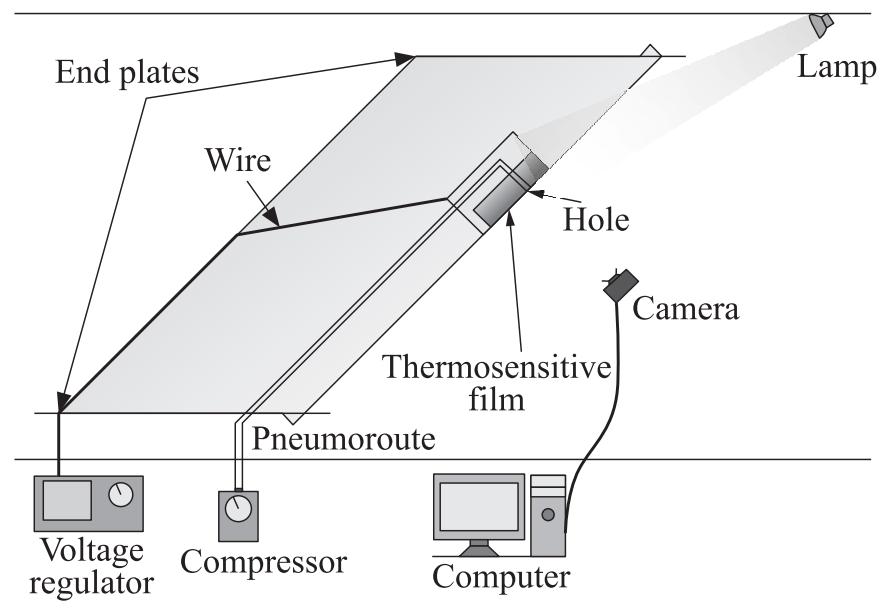

Figure 3 Experimental setup for investigating vortex structure on the oblique wing leading edge by LCT

In an aerodynamic experiment, liquid crystals can be used for visualization of temperature and shear stress distributions [16]. In the present experiments, liquid-crystal thin-film coatings based on cholesteric liquid crystals insensitive to shear stresses were used. The method is based on the dependence of wavelength selective reflection of the cholesteric on the temperature. As a result, the color of the film will depend on the temperature and observation angles. With increasing temperature, the coating changes color from brown-red to blue-purple. The temperature distribution on the examined surface can be obtained using temperature-color and angle-color calibrations. The film has a certain temperature range $\Delta T$, beyond of which the coating loses the property of selective reflection. With reducing the operating range, the film becomes more sensitive. Thus, it is necessary to find the correct parameters of the film. In these experiments, the operating range of the film was $\Delta T=3{ }^{\circ} \mathrm{C}$.

To study the quasi-stationary flow structure near the wall close to the leading edge of the oblique wing (Fig. 3), constant heat flux boundary conditions were implemented by an ohmic heater. A layer of liquid crystal film was fixed on the top of the heater (Fig. 4).

Ohmic heater power is regulated by dimmer to place the film temperature in the operating range. In [17], it was found that the overheating of the surface at $10-15{ }^{\circ} \mathrm{C}$ does not have significant influence on transversal scale of the vortex structures. In the present experiment, a good agreement between LCT and hotwire anemometry was found. However, it is important to minimize the heating to reduce its affect on the flow structure. 


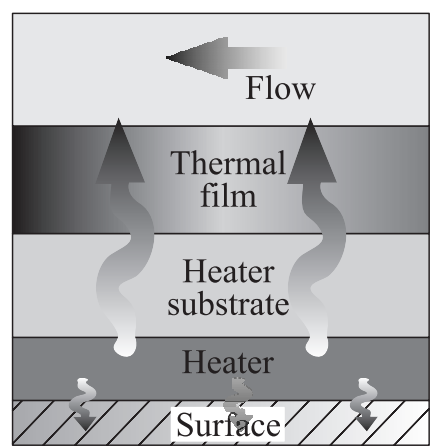

(a)

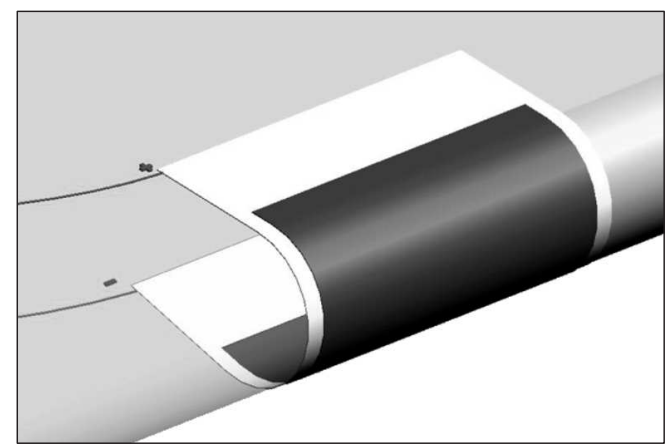

(b)

Figure 4 Schematic layout of temperature-sensitive coating $(a)$ and its position at the leading edge of the oblique wing $(b)$

Since the specific heat capacity is constant, the increase in heat transfer leads to decrease of the liquid-crystal-film temperature. The resulting color distribution of the liquid crystal coating was recorded by digital camera and transmitted to the computer.

The camera position was chosen to minimize the angular dependence of the color of liquid-crystal film, to maximize the visualization area, and not to affect the flow under investigation.

It is necessary to highlight some features of LCT:

- informative rapid diagnosis and reusable;

- high spatial resolution and temperature sensitivity;

- a model should be made of a heat insulator or there should be thermal isolation on the model of metal to avoid the spreading of heat;

- in a subsonic flow with small temperature variations, one needs a quality ohmic heater with a uniform distribution of heat power. Nonuniformity of heat flux can be controlled by the liquid-crystal coating color in preliminary tests without flow;

- there is a need of a quality digital camera for accurate film color calibration in the experimental conditions to obtain quantitative data on the temperature;

- time to reach a steady temperature distribution on the wing is about 20$30 \mathrm{~min}$; 
- the cholesteric liquid crystals response time on the step heat exposure is from 30 to $200 \mathrm{~ms}$ depending on the composition [18]; and

- using the method one can resolve the stationary structure of flow disturbance.

Despite the limitations, the technique of LCT is a very convenient tool for the qualitative analysis of stationary structure of the near-wall flow.

Processing of visualization data which will be further referred to as the method of subtraction, consisted of four stages. First, trim unwanted information (areas that lie outside the field of measurement). To highlight the flow disturbance, the difference between perturbed and unperturbed flow patterns was calculated. The resulting data were processed by a median filter with a window $3 \times 3$ pixels to remove high-frequency noise. The final stage was carrying out the normalization of brightness.

\section{GENERATION AND FIRST STAGES OF DEVELOPING OF LONGITUDINAL VORTEX STRUCTURES}

\subsection{Conditions for Generation and Development of Disturbance}

Blowing through a hole at the leading edge of the wing forms a stationary longitudinal vortex disturbance. The air jet in such conditions works as an obstacle for the flow; so, it is a certain analogy with the roughness. Depending on the relative position of the hole for blowing and attachment line, disturbance is propagated on one or other surface of the wing. Adjusting blowing velocity one can change the amplitude and behavior of the longitudinal disturbance, in particular, the propagation path. It is because of highly three-dimensional structure near the attachment line; so, depending on the strength of blowing, the air jet reaches the different height from the surface, where the velocity vector changes the direction, thus the disturbances propagated in different directions, at least in the initial stage of development. Variation of blowing velocity has an influence on the vorticity intensity. There were considered two regimes of injection: $U / U_{0}=5 \%$ (small amplitude) and $U / U_{0}=8 \%$ (large amplitude) (Fig. 5). Despite a slight difference between the amplitudes of the perturbations, they are qualitatively different. The relative position between the hole for blowing and attachment line was adjusted to provide maximal path of the disturbance propagation along the attachment line.

To determine the flow conditions, hot-wire measurements were carried out on the oblique wing leading edge boundary layer on the attachment line (Fig. 6). Analysis of the velocity profiles showed the constant boundary-layer thickness 


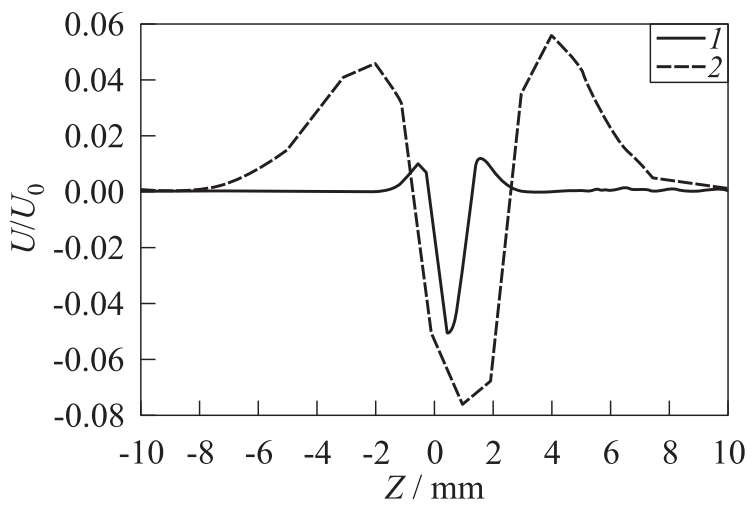

Figure 5 Velocity disturbance distributions at 5-millimeter distance from the blowing location for small (1) and high (2) amplitude regimes (weak and strong blowing, respectively)

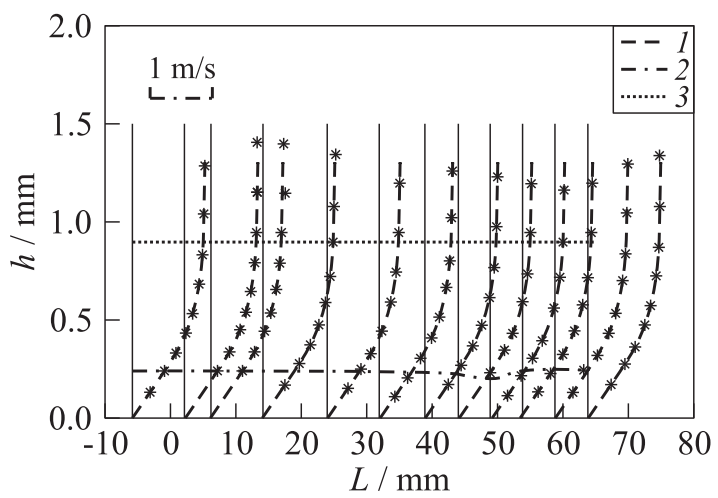

Figure 6 Velocity profiles (1), displacement thickness (2), and 0.99 boundary layer thickness (3) on the attachment line of oblique wing

on the attachment line, the 99 percent level was $\delta=0.9 \mathrm{~mm}$. The external-flow velocity was $U_{0}=3.5 \mathrm{~m} / \mathrm{s}$. In these conditions, the Reynolds number calculated using the boundary layer displacement thickness was $\mathrm{Re}=58.6$.

\subsection{Small Amplitude Regime}

The next step was pneumoroute adjusting in small amplitude regime to highlight artificial velocity disturbances from the background noise in conditions of minimal stimulation. This setup provided velocity defect equal to $5 \%$ of the 


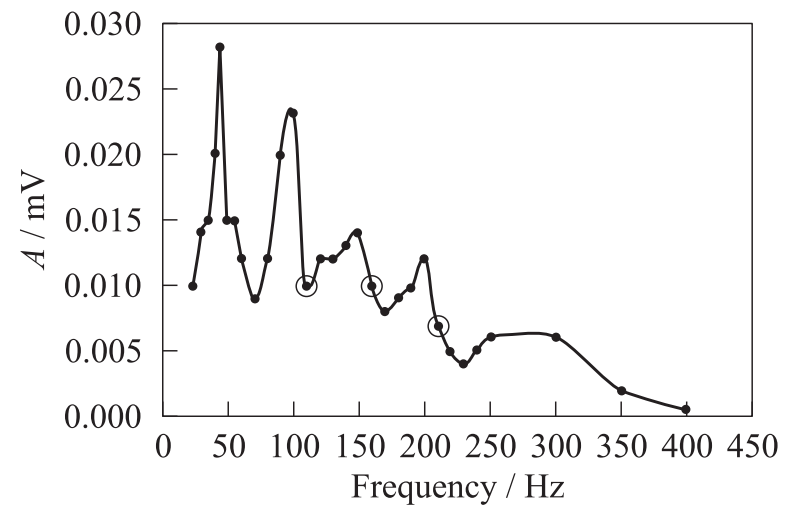

Figure 7 Frequency response of the compressor-pneumoroute system on 5-millimeter distance from the source of disturbances

undisturbed velocity at 5-millimeter distance along the attachment line from the blowing location. After that, high-frequency disturbances of different frequencies were introduced jointly with stationary disturbance and the frequency response of the compressor-pneumoroute system was investigated (Fig. 7).

The frequencies that are multiples of 50-hertz allowed maximum, but in terms of noise, these frequencies are unfavorable, because all devices are powered by a 50-hertz electrical source, including a compressor. For the further measurements, several frequencies separated from the resonance, that is, 110, 160 , and $210 \mathrm{~Hz}$ were selected.

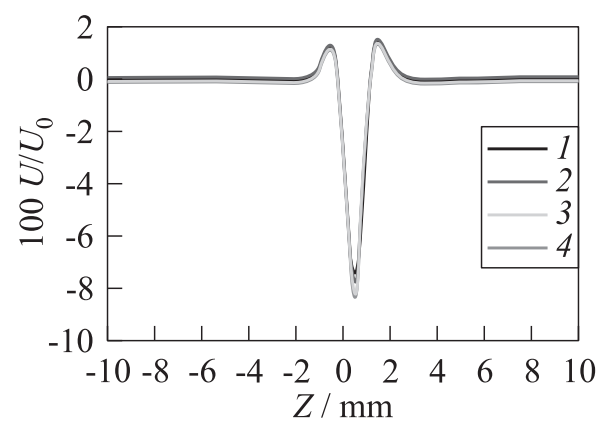

(a)

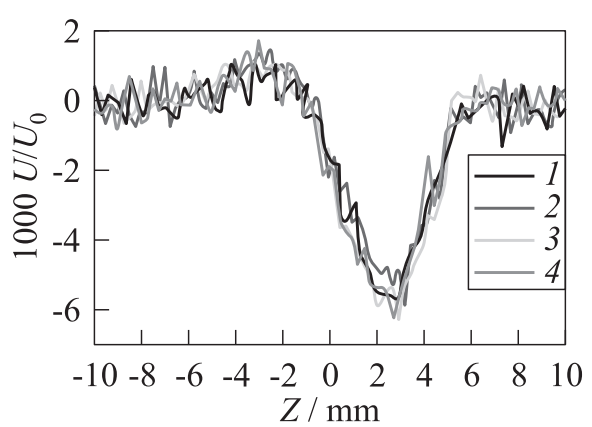

(b)

Figure 8 Velocity disturbance distributions for regimes with different frequencies of injected perturbations $(1-0 \mathrm{~Hz} ; 2-110 ; 3-160$; and $4-210 \mathrm{~Hz})$ on 5 -millimeter $(a)$ and 25-millimeter $(b)$ distances from the blowing source 
The results of hot-wire measurements showed the absence of influence of disturbances with the above frequencies on the mean flow (Fig. 8). Thus, these disturbances were the linear ones.

For a more detailed analysis of behavior of the quasi-stationary and highfrequency disturbances, hot-wire measurements were carried out on the grid parallel to the $Z$ axis and the leading edge of the swept wing, with and without superposition of the high-frequency disturbances. Through data processing, the distributions of the stationary velocity disturbance (Fig. 9), the amplitude of the high frequency disturbance (Fig. 10), the standard deviation of the natural (Fig. 11), and artificial high-frequency disturbances were obtained.

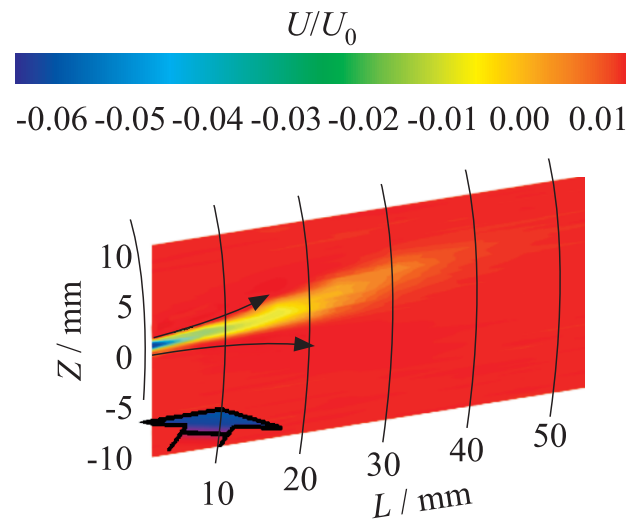

Figure 9 Velocity disturbance distribution in small amplitude regime

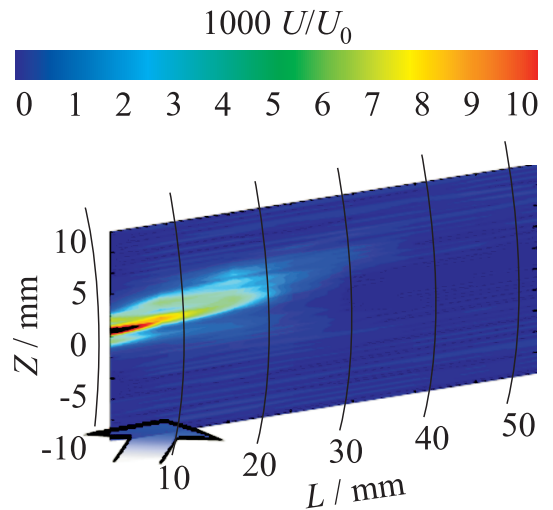

(a)

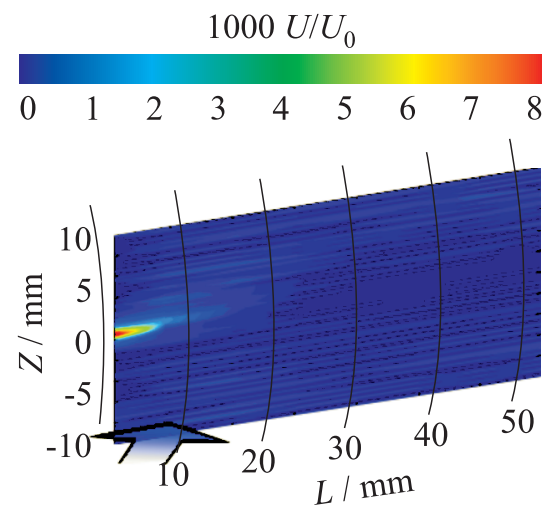

(b)

Figure 10 High-frequency disturbance amplitude distribution in superposition with $(a)$ and without $(b)$ stationary mode 


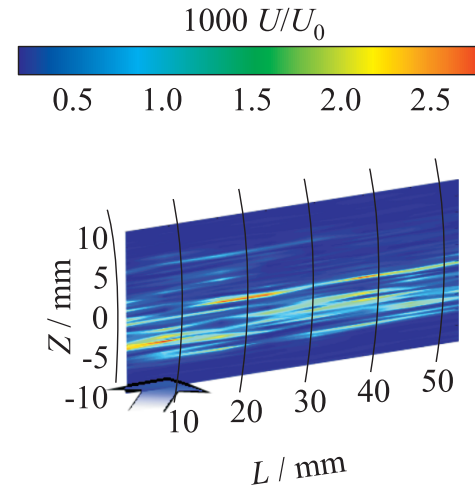

(a)

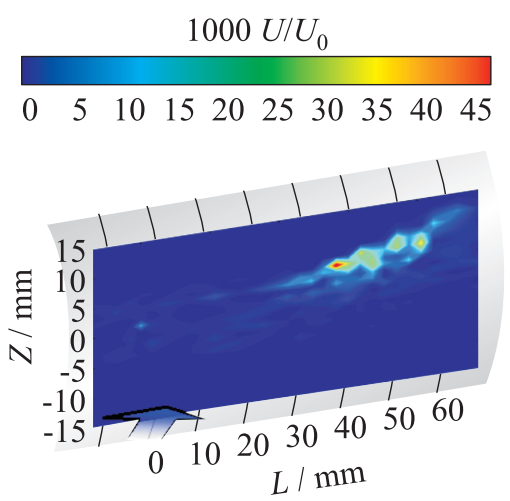

(b)

Figure 11 Distribution of velocity pulsations in small (a) and high (b) amplitude regimes of the excited stationary disturbance

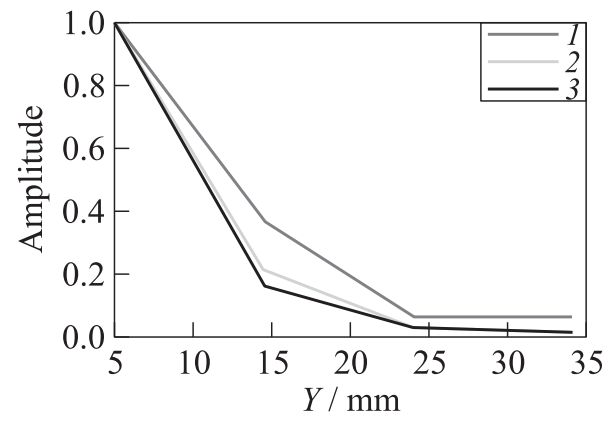

(a)

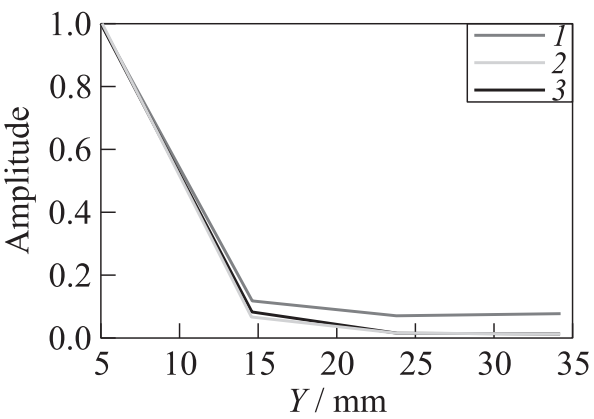

(b)

Figure 12 High-frequency disturbance decaying along the oblique wing leading edge superposed with $(a)$ and without $(b)$ stationary mode: $1-110 \mathrm{~Hz} ; 2-160$; and $3-$ $210 \mathrm{~Hz}$

Analysis of the stationary velocity disturbance showed that in current experimental conditions, the stationary vortices propagated on both sides from the attachment line (see Fig. 9). There was observed a redistribution of energy between two vortices in accordance with the relative positions of disturbance source and attachment line. The stationary disturbances did not grow along the propagation direction.

Introduced high-frequency disturbances decayed rapidly. It was found that presence of stationary vortices leads to decrease of boundary layer stability (see Figs. 10 and 12); so, the location of high-frequency disturbances was correlated 
with the location of stationary vortices (see Fig. 10a). Comparative analysis of the decay rate revealed the dependence of this value from the frequency of disturbance (see Fig. 12).

Character of natural pulsations distribution at small amplitude stationary disturbance regime (see Fig. 11a) did not reveal the special role of stationary disturbances on the formation of secondary instability and the growth of the natural fluctuations was not observed.

\subsection{High-Amplitude Regime}

The next step was adjusting pneumoroute to the large amplitude regime. Velocity defect at a distance of $5 \mathrm{~mm}$ from the source of disturbance was $8 \%$ (see Fig. 5) of the along the leading edge velocity component. The transverse size of the vortex disturbance is three times the size of the small amplitude vortex. Hot-wire measurements were carried out on the flat grid (see Fig. $2 a$ ) and in the volume (see Fig. $2 b$ ) formed by seven planes as described in subsection 2.4. After processing the data, the velocity disturbance distribution (Fig. 13), velocity pulsations in the plane and in the volume (Fig. 14) were plotted.

Analysis of the positive and negative velocity deviations showed that the stationary vortices propagated along the boundary layer flow (see Fig. 13). The velocity disturbance distribution indicated the presence of two counterrotating longitudinal vortices, one of which began to dominate over another one in downstream direction (see Fig. 14).

The velocity pulsation distribution character (see Fig. 11b) shows a presence of favorable conditions for natural disturbance development and appearance of secondary instability in the flow region, disturbed by the stationary mode.

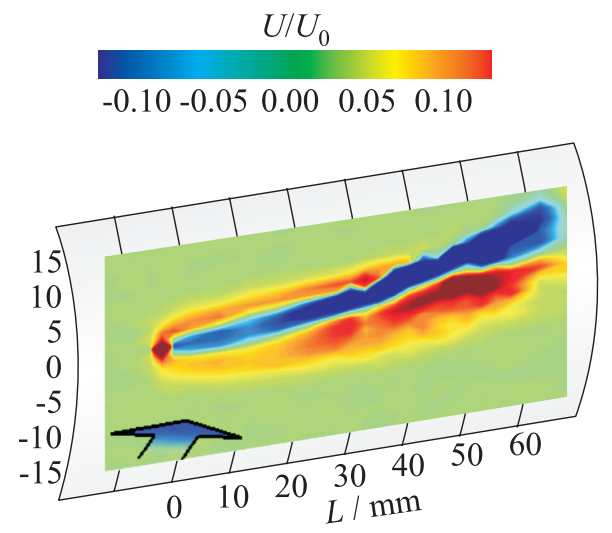

Figure 13 Velocity disturbance distribution in high-amplitude regime 

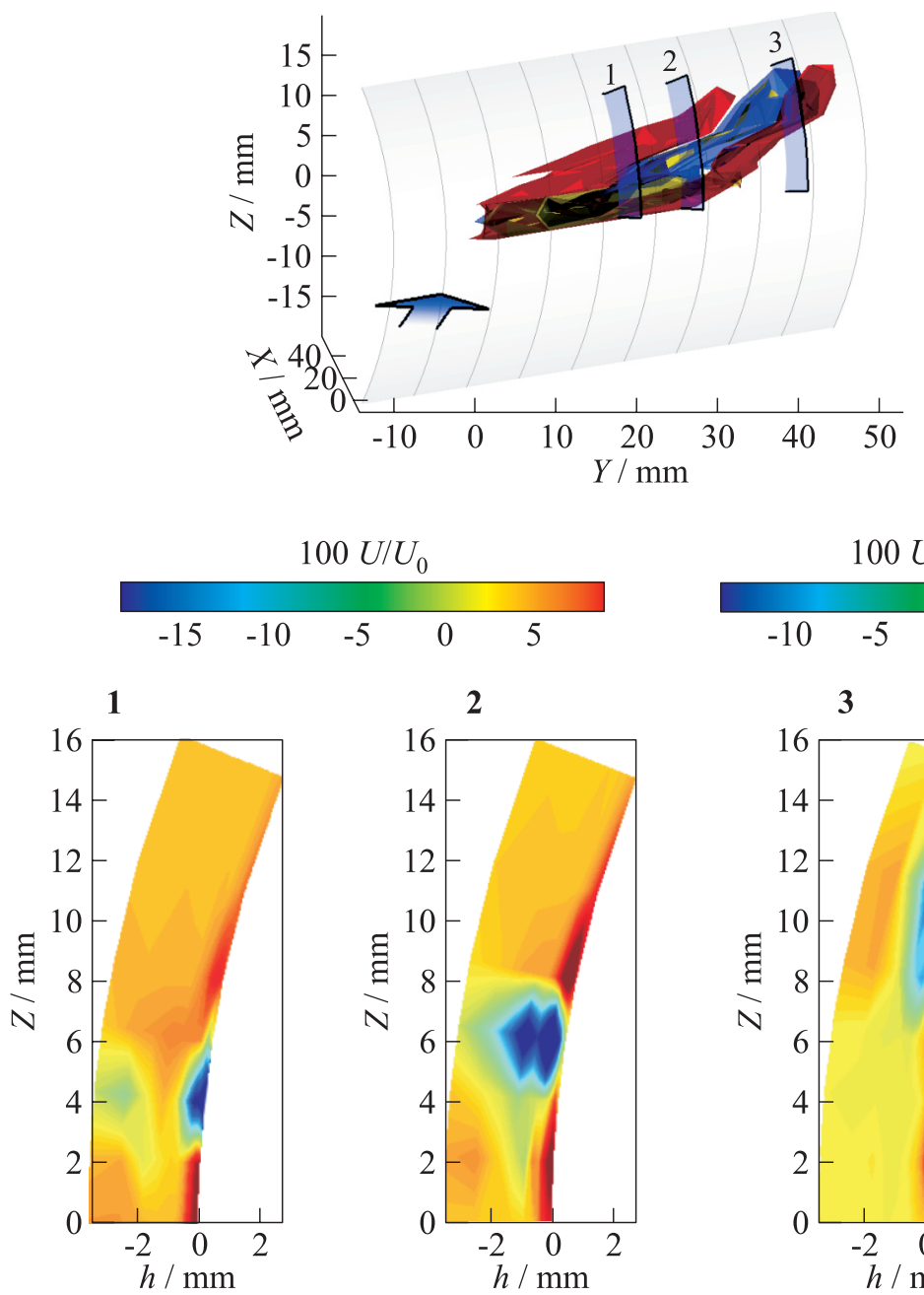

$100 U / U_{0}$
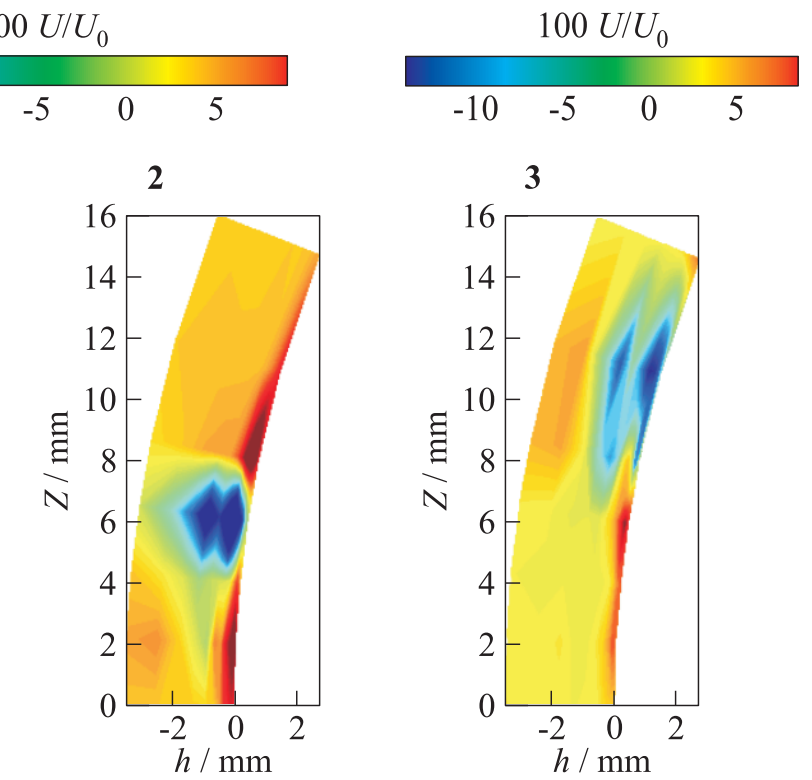

Figure 14 Isosurfaces of positive $6.3 \cdot 10^{-3} U_{0}$ (red) and negative $3.5 \cdot 10^{-2} U_{0}$ (blue) deviations of mean velocity, isosurface of root-mean-square values of velocity perturbations $7.9 \cdot 10^{-3} U_{0}$ (yellow) and velocity disturbance distributions in three sections $1-3$

\subsection{Liquid Crystal Thermography Visualization Pictures}

Changing the point of disturbance injection relative to the attachment line led to a change in the trajectory of stationary disturbance (Fig. 15). The increase of angle between disturbance source and attachment line led to the increase of 


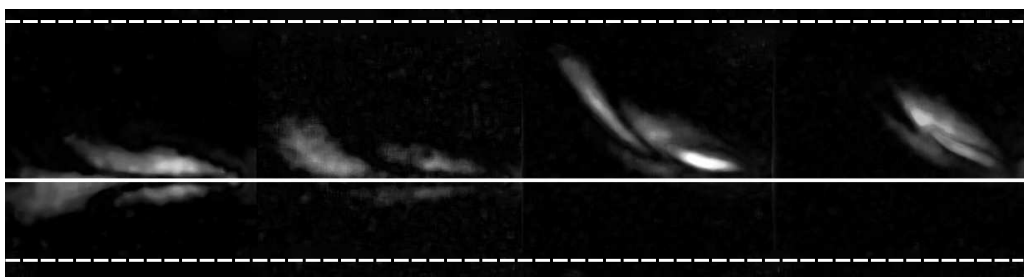

(a)

(b)

(c)

(d)

Figure 15 Stationary disturbance's trajectories for different blowing locations relative to the attachment line (white line): $(a) \alpha=-0.16^{\circ}$ and $d=-0.1 \mathrm{~mm} ;(b) \alpha=0.16^{\circ}$ and $d=0.1 \mathrm{~mm} ;(c) \alpha=1.13^{\circ}$ and $d=0.8 \mathrm{~mm}$; and $(d) \alpha=2.59^{\circ}$ and $d=1.8 \mathrm{~mm}$. The free-stream velocity was $3.4 \mathrm{~m} / \mathrm{s}$. Dashed lines are the boundaries of cylinder which define the leading edge in frontal view

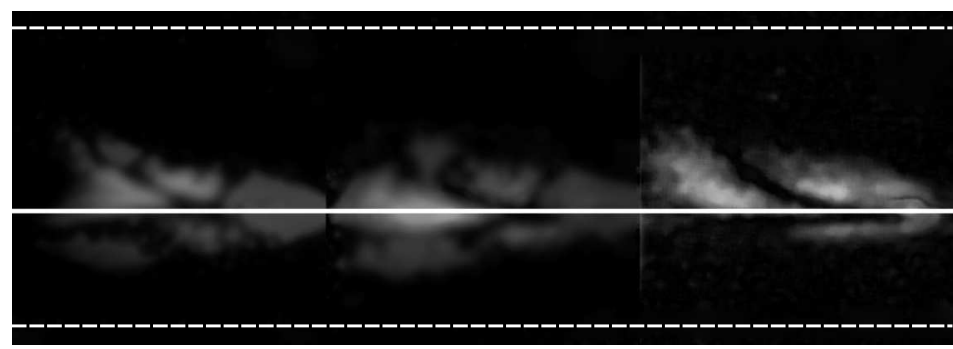

(a)

(b)

(c)

Figure 16 Sliding of the stationary vortices when blowing source location is near the attachment line (white line). The free-stream velocity is $3.4 \mathrm{~m} / \mathrm{s}$. Dashed lines are the boundaries of the cylinder which define the leading edge in frontal view

angle between the initial trajectory of the disturbance and attachment line and had lower transverse dimension of stationary vortices. The first feature has an explanation that resulting vortex structures were the longitudinal ones.

In the case of closeness of blowing point to the attachment line, there was a chaotic vortex sliding in one (Fig. 16a) or another side (Figs. $16 b$ and 16c). To avoid previous effect, the blowing point was offset from the attachment line. The next step was to study the influence of the blowing velocity on the stationary mode behavior.

Increasing the blowing speed (Fig. 17) increased the amplitude of the stationary disturbances, its transverse dimension, and changed a little its trajectory. An angle between the disturbance trajectory and the attachment line became smaller that could be caused by the interaction between the stationary vortices and the 


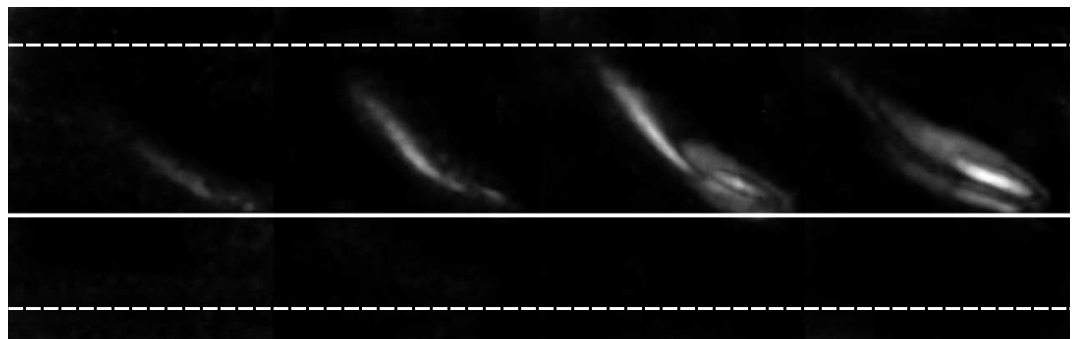

(a)

(b)

(c)

(d)

Figure 17 Stationary mode behavior depending on the blowing velocity: (a) $0.8 \mathrm{~m} / \mathrm{s}$; (b) $1.1 ;(c) 2.5$; and $(d) 13.8 \mathrm{~m} / \mathrm{s}$. Blowing is at $1.24 \mathrm{~mm}$ from the attachment line (white line). The free-stream velocity is $3.4 \mathrm{~m} / \mathrm{s}$. Dashed lines are the boundaries of the cylinder which define the leading edge in frontal view

surface.

In one of the blowing regimes, multiplication of stationary vortices was observed (Fig. 18). Instead of two counterrotating vortices, four of them were found. There are two explanations of this phenomenon. The first is that air jet from the hole interacts with the oncoming flow in the boundary layer in complex way and excites four vortices instead of two. This situation is not typical for the flow after roughness element. The second explanation is that the trajectory of the pair of stationary disturbances is very sensitive to the uncontrolled blowing

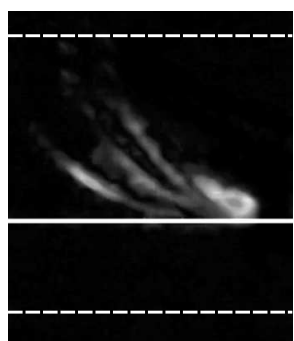

Figure 18 Multiplication of stationary vortices. Blowing is at $1.24 \mathrm{~mm}$ from the attachment line (white line). Blowing velocity is $11.6 \mathrm{~m} / \mathrm{s}$. The freestream velocity is $9.4 \mathrm{~m} / \mathrm{s}$. Dashed lines are the boundaries of the cylinder which define the leading edge in frontal view

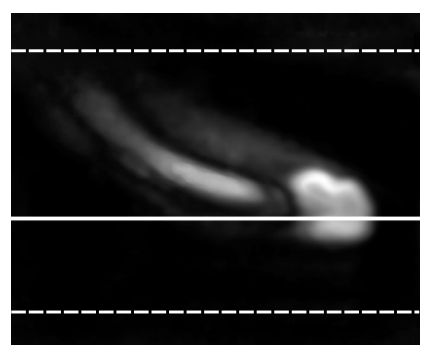

Figure 19 Highly nonlinear stationary structure for blowing at $1.24 \mathrm{~mm}$ from the attachment line (white line). Blowing velocity is higher than $16 \mathrm{~m} / \mathrm{s}$. The freestream velocity is $3.4 \mathrm{~m} / \mathrm{s}$. Dashed lines are the boundaries of the cylinder which define the leading edge in frontal view 
velocity variations in the compressor, creating the illusion of four vortices at the visualization.

Here, it will be highlighted the strong blowing regime (Fig. 19) in which the compressor was running at full power, but the jet velocity through the hole is unknown because the flowmeter was not designed for such flow rate $\left(V_{\text {jet }} \gg 16 \mathrm{~m} / \mathrm{s}\right)$. At visualization, a wide disturbance "tail" caused by nonlinear processes in the modified flow was observed.

\section{CONCLUDING REMARKS}

\subsection{Hot-Wire Anemometry}

Investigation of the flow structure at the leading edge of the oblique wing using controlled disturbances excitation in two blowing regimes was carried out. A technique of hot-wire measurement over the curved surface was developed. Hot-wire measurements showed a constant boundary layer thickness along the attachment line which allowed not to take into account the end effects in the measurement domain.

Spatial stationary disturbance distribution shows the existence of two counterrotating longitudinal vortices. The stationary mode behavior depended on the velocity defect created by blowing through the small hole. In the regime of weak blowing, there was no stationary disturbance amplitude growing. Investigations of linear high-frequency disturbances and their interaction with stationary mode showed decrease of their amplitude along the stream and lower decay rate in the regime of superposition with the stationary mode. The increase of disturbance frequency increased its decay rate. In high-velocity blowing regime, the stationary disturbance, secondary oscillations, and the natural pulsations increased their amplitude along the stationary vortex.

\subsection{Liquid Crystal Thermography Technique}

Results of LCT visualization are in a good agreement with the hot-wire measurements. The stationary local blowing in the boundary layer generates a pair of counterrotating vortices with their amplitude and transverse scale dependent on the blowing rate.

Features of LCT allowed to receive visualization pictures in a wide range of adjustable parameters and to define the influence of the injection velocity on the stationary disturbance trajectory - with increasing injection velocity, the angle between the trajectory of disturbance and the attachment line decreases, which may be due to the interaction of the stationary vortex with the wall. There is 
observed the instability of stationary disturbance trajectory when the source of the disturbance is located close to the attachment line - one of the vortices is travelled from one side from the attachment line to another. In one regime of blowing, on the visualization pattern, four tracks from stationary disturbances were found, which showed complex interaction of the air jet with the incoming flow in boundary-layer conditions. Very strong blowing led to the formation of a wide disturbance, size of which was almost unchanging, indicating the highly nonlinear character.

Common result of the work is that weak blowing can be used as the tool for the boundary-layer investigation at the leading edge of the oblique wing. Strong blowing leads to complicate character of the flow and appearance of strong nonlinear effects and could be investigated separately, results of which could be used for the flow control and solving ice formation problems.

\section{ACKNOWLEDGMENTS}

This work was supported by the Russian Foundation for Basic Research, grant No. 12-01-31347.

\section{REFERENCES}

1. Blackwelder, R.F. 1983. Analogies between transitional and turbulent boundary layers. Phys. Fluids 27(6):1345-1347.

2. Reed, H. L., and W. S. Saric. 1989. Stability of three-dimensional boundary layers. Ann. Rev. Fluid Mech. 21:235-284.

3. Orszag, S. A., and A.T. Patera. 1983. Secondary instability of wall-bounded shear flows. J. Fluid Mech. 128:347-385.

4. Zhigulyev, V.N., and A.M. Tumin. 1987. Generation of the turbulence. Dynamic theory of generation and development of instability in the boundary layers. Novosibirsk: Nauka. 109-114. [In Russian.]

5. Kohama, Y. 1987. Some expectation on the mechanism of cross-flow instability in a swept-wing flow. Acta Mech. 66:21-38.

6. Poll, D. I. A. 1985. Some observations on the transition process on the windward face of a long yawed cylinder. J. Fluid Mech. 30:329-356.

7. Nishizawa, A., N. Tokugawa, and S. Takagi. 2009. Experimental investigation of the flow instability near the attachment-line boundary layer on a yawed cylinder. Fluid Dyn. Res. 41:035513.

8. Zharkova, G. M., and A.S. Sonin. 1994. Liquid-crystal composites. Novosibirsk: Nauka, Siberian branch. 214 p. [In Russian.] 
9. Zharkova, G. M., V.N. Kovrizhina, and V. M. Khachaturyan. 2002. Experimental study of subsonic flows by liquid-crystal thermography. J. Appl. Mech. Tech. Phys. 43(2):274-279.

10. New, T. H., T. T. Lim, and S. C. Luo. 2006. Effects of jet velocity profiles on a round jet in cross-flow. Exp. Fluids 40(3):859-875.

11. Bagheri, S., Ph. Schlatter, P. J. Schmid, and D. S. Henningson. 2009. Global stability of jet in cross-flow. J. Fluid Mech. 624:33-44.

12. Kozlov, V. V., G. R. Grek, M. V. Litvinenko, Yu. A. Litvinenko, and G. V. Kozlov. 2010. Round jet in transversal shear flow (review). Vestnik NSU. Ser. Phys. 5(1):9. [In Russian.]

13. Bakchinov, A. A., K. J. A. Westin, V. V. Kozlov, and P. H. Alfredsson. 1998. Experiments on localized disturbances in a flat plate boundary layer. Part 2. Interaction between localized disturbances and TS-waves. Eur. J. Mech. B Fluid. 17:847-873.

14. Bakchinov, A. A., G. R. Grek, M. M. Katasonov, and V. V. Kozlov. 1998. Experimental investigation of the interaction of longitudinal streaky structures with a high-frequency disturbance. Fluid Dyn. 33(5):667-675.

15. Grek, G. R., M. M. Katasonov, V. V. Kozlov, and V. G. Chernoray. 1999. Modeling of "puff" - structures in two- and three dimensional boundary layers. Novosibirsk: ITAM. Preprint No. 2-99. [In Russian.]

16. Zharkova, G. M., V.N. Kovrizhina, A.P. Petrov, E. S. Shapoval, V.E. Mosharov, and V.N. Radchenko. 2011. Visualization of boundary layer transition by shear sensitive liquid crystals. 8th Pacific Symposium on Flow Visualization and Image Processing PSFVIP-8 Proceedings. Moscow, Russia. 113:1-5.

17. Brylyakov, A. P., G. M. Zharkova, B. Yu. Zanin, V. N. Kovrizhina, and D. S. Sboyev. 2004. Effect of free-stream turbulence on the flow structure near a wedge and the windward side of an airfoil. J. Appl. Mech. Tech. Phys. 45(4):510-516.

18. Ireland, P. T., and T. V. Jones. 1987. The response time of a surface thermometer employing encapsulated thermochromic liquid crystals. J. Phys. E Sci. Instrum. 20:1195 